\title{
CHIPS: A Snakemake pipeline for quality control and reproducible processing of chromatin profiling data [version
}

\section{1; peer review: 1 not approved]}

\author{
Len Taing 1,2*, Gali Bai (D1) ${ }^{*}$, Clara Cousins ${ }^{1 *}$, Paloma Cejas², Xintao Qiu2, \\ Zachary T. Herbert ${ }^{3}$, Myles Brown ${ }^{2,4}$, Clifford A. Meyer ${ }^{1,5}$, X. Shirley Liu'1,2,5, \\ Henry W. Long (i) 2 , Ming Tang ${ }^{1}$ \\ ${ }^{1}$ Department of Data Science, Dana-Farber Cancer Institute, Boston, MA, 02215, USA \\ ${ }^{2}$ Center for Functional Cancer Epigenetics, Dana-Farber Cancer Institute, Boston, MA, 02215, USA \\ ${ }^{3}$ Molecular Biology Core Facilities, Dana-Farber Cancer Institute, Boston, MA, 02215, USA \\ ${ }^{4}$ Department of Medical Oncology, Dana-Farber Cancer Institute, Boston, MA, 02215, USA \\ ${ }^{5}$ Department of Biostatistics, Harvard T.H. Chan School of Public Health, Boston, MA, 02215, USA \\ * Equal contributors
}

V1 First published: 30 Jun 2021, 10:517

https://doi.org/10.12688/f1000research.52878.1

Latest published: 30 Jun 2021, 10:517

https://doi.org/10.12688/f1000research.52878.1

\section{Abstract}

Motivation: The chromatin profile measured by ATAC-seq, ChIP-seq, or DNase-seq experiments can identify genomic regions critical in regulating gene expression and provide insights on biological processes such as diseases and development. However, quality control and processing chromatin profiling data involves many steps, and different bioinformatics tools are used at each step. It can be challenging to manage the analysis.

Results: We developed a Snakemake pipeline called CHIPS (CHromatin enrIchment ProcesSor) to streamline the processing of ChIP-seq, ATAC-seq, and DNase-seq data. The pipeline supports single- and paired-end data and is flexible to start with FASTQ or BAM files. It includes basic steps such as read trimming, mapping, and peak calling. In addition, it calculates quality control metrics such as contamination profiles, polymerase chain reaction bottleneck coefficient, the fraction of reads in peaks, percentage of peaks overlapping with the union of public DNaseI hypersensitivity sites, and conservation profile of the peaks. For downstream analysis, it carries out peak annotations, motif finding, and regulatory potential calculation for all genes. The pipeline ensures that the processing is robust and reproducible.

Availability: CHIPS is available at https://github.com/liulabdfci/CHIPS.

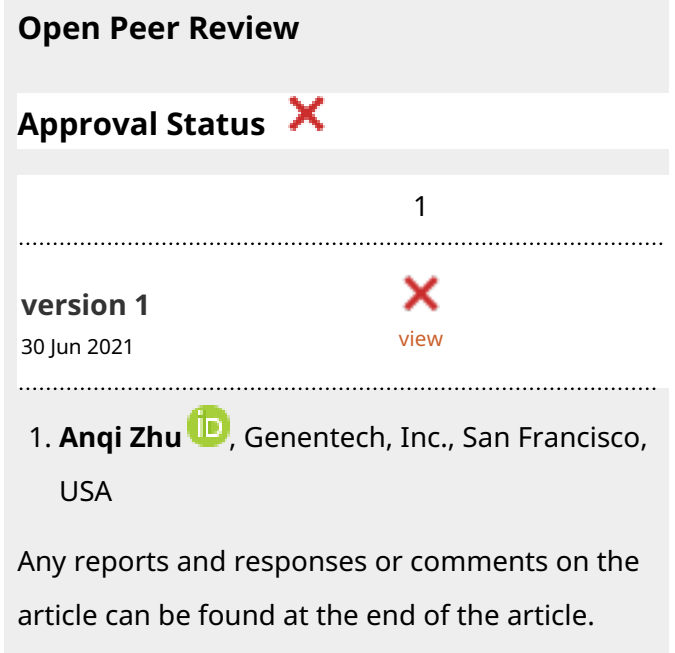


Keywords

chromatin profiling, snakemake, ChIP-seq, ATAC-seq

Corresponding authors: Henry W. Long (HENRY_LONG@dfci.harvard.edu), Ming Tang (mtang@ds.dfci.harvard.edu)

Author roles: Taing L: Software, Writing - Original Draft Preparation, Writing - Review \& Editing; Bai G: Software, Writing - Original Draft Preparation, Writing - Review \& Editing; Cousins C: Software, Writing - Original Draft Preparation, Writing - Review \& Editing; Cejas P: Resources, Validation, Writing - Review \& Editing; Qiu X: Resources, Validation, Writing - Review \& Editing; Herbert ZT: Resources, Validation, Writing - Review \& Editing; Brown M: Resources, Validation, Writing - Review \& Editing; Meyer CA: Resources, Validation, Writing - Review \& Editing; Liu XS: Funding Acquisition, Supervision, Validation, Writing - Review \& Editing; Long HW:

Resources, Software, Supervision, Validation, Writing - Review \& Editing; Tang M: Software, Supervision, Validation, Writing - Original Draft Preparation, Writing - Review \& Editing

Competing interests: No competing interests were disclosed.

Grant information: This work has been supported by grants from the National Institute of Health (U24CA237617 and U24CA224316). HWL acknowledges support from National Institute of Health grants 2PO1CA163227 and P01CA080111.

The funders had no role in study design, data collection and analysis, decision to publish, or preparation of the manuscript.

Copyright: @ 2021 Taing L et al. This is an open access article distributed under the terms of the Creative Commons Attribution License, which permits unrestricted use, distribution, and reproduction in any medium, provided the original work is properly cited.

How to cite this article: Taing L, Bai G, Cousins C et al. CHIPS: A Snakemake pipeline for quality control and reproducible processing of chromatin profiling data [version 1; peer review: 1 not approved] F1000Research 2021, 10:517

https://doi.org/10.12688/f1000research.52878.1

First published: 30 Jun 2021, 10:517 https://doi.org/10.12688/f1000research.52878.1 


\section{Introduction}

Protein-DNA binding interactions are fundamental to gene regulation and are involved in regulating disease processes. However, the methods of investigating these interactions through ATAC-seq, ChIP-seq, and DNase-seq experiments generate data that require extensive processing before biological interpretation (Furey 2012). Chromatin profiling using sequencing technology can also generate bias (Meyer and Liu 2014), which needs to be mitigated before interpreting the biological significance. Therefore, consistent and reproducible processing of the chromatin profiling data is essential in deriving meaningful information from the experimental data. Moreover, experiments can fail due to technical complexities. Comprehensive quality control will help to identify failed samples, and robust processing can facilitate reproducible analysis.

There are other pipelines available for processing ChIP-seq data. For example, ENCODE has its own pipeline written in Workflow Description Language (WDL) (The ENCODE Project Consortium 2012). Recently, Snakemake (Köster and Rahmann 2012) workflow language becomes popular in the bioinformatics field partly because it is python-based. Using Snakemake v5.4.5 we developed CHromatin enrIchment ProcesSor (CHIPS) to standardize processing and quality control evaluation for ATAC-seq, ChIP-seq, and DNase-seq data following best practice (Bailey et al. 2013). Furthermore, CHIPS generates a comprehensive interactive HTML report using Plotly for the users to easily inspect the quality of the samples. Encapsulated in a Conda environment, it can be executed in the local computing cluster engine or in the cloud computing settings such as Amazon AWS and Google Cloud. CHIPS has been used to analyze $>1500$ samples since 2016 within Dana-Farber Cancer Institute, and now serves as the standard processing pipeline for tumor ATAC-seq data from the Cancer immune Monitoring and Analysis Centers and Cancer Immunologic Data Commons (CIMACCIDC) trials (H. X. Chen et al. 2021).

\section{Methods}

Implementation

Alignment and basic quality control

The workflow of CHIPS is described in Figure 1. CHIPS takes FASTQ or BAM files as input and supports both singleend and paired-end data. To save time and resources, CHIPS subsamples 100,000 reads and uses them in the FASTQC

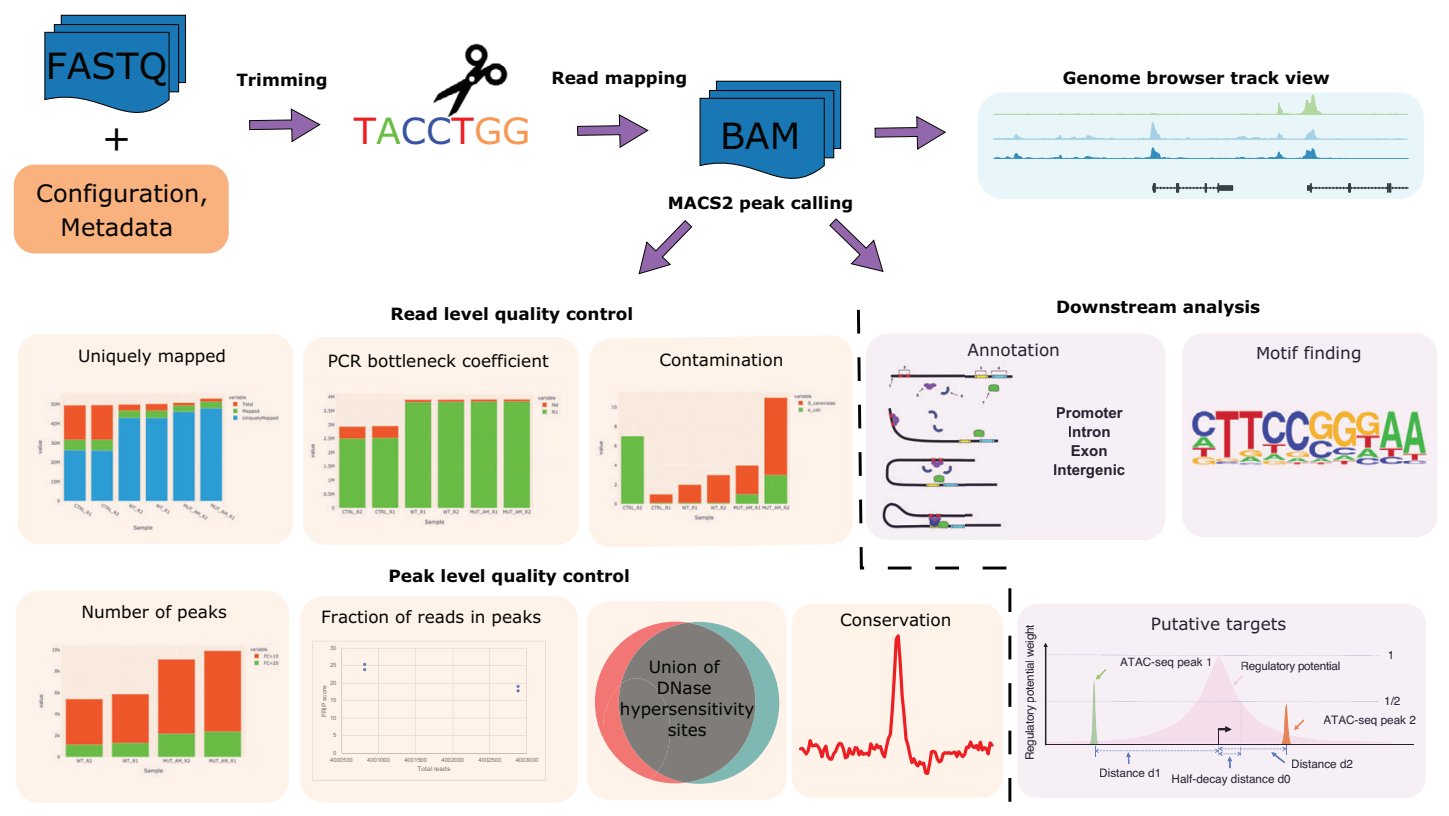

Figure 1. CHromatin enrIchment ProcesSor (CHIPS) workflow. The CHIPS pipeline is designed to perform robust quality control and reproducible processing of chromatin profiling data derived from ChIP-seq, ATAC-seq, and DNase-seq. The CHIPS pipeline includes basic steps of read trimming, read alignment, and peak calling. For quality control, it calculates metrics such as contamination profile, mapping statistics, the fraction of reads in peaks (FRIP) score, PCR bottleneck coefficient (PBC), overlap with union DNaseI hypersensitive sites (DHS), and peak evolutionary conservation. For downstream analysis, CHIPS carries out peak annotation, motif finding, and putative target prediction. The inputs to the pipeline are FASTQ/BAM format DNA sequence read files. 
module for basic quality control analysis. For aligning reads to the reference genome, FASTQ files are trimmed to remove adaptors and low-quality sequences using fastp (S. Chen et al. 2018) and then aligned by BWA-MEM (Li 2013) to generate sorted and deduplicated BAM files. After alignment, the mapping statistics, including the number of mapped and uniquely mapped reads, are then reported.

CHIPS carries out other basic quality control. The contamination profile reports the percentage of 100,000 reads that map to a contamination panel's reference genomes. The contamination panel, specified by the user in a configuration file, includes dm3, Saccharomyces cerevisiae, E. coli, and mycoplasma of different types in addition to hg38, hg19, $\mathrm{mm} 10$, and $\mathrm{mm} 9$ genomes. We provide static reference files along with the installation of CHIPS. Users may add new assemblies to the contamination panel by adding the BWA index files. In addition, 4,000,000 reads are downsampled for calculating the PCR bottleneck coefficient (PBC). The PBC is the number of locations with exactly one uniquely mapped read divided by the number of uniquely mapped genomic locations. PBC ranges from $0-1$, and a higher number indicates higher library complexity.

Particularly useful in the setting of ATAC-seq experiments, CHIPS also provides a fragment lengths distribution plot. ATAC-seq data with high quality should have fragment length peaks at $<100$ bp nucleosome-free regions and show periodical enrichment at the 1- and 2-nucleosome lengths.

Peak calling and peak characteristics for quality control

Peaks represent regions of the genome that are enriched with aligned reads. The MACS2 (Zhang et al. 2008) algorithm is used to call peaks from uniquely sorted BAM files. The minimum false discovery rate (FDR) cutoff for defining peak confidence is set to 0.01 by default but can be changed in the config.yaml file. A summary of the number of peaks, including those with a $>10$ or $>20$-fold increase relative to the background, is also reported describing the data quality. More peaks and a higher fraction of $>10 \mathrm{X}$ peaks tend to indicate higher quality. Moreover, a read per million (RPM) normalized BedGraph signal track file generated by MACS2 is further converted to a BigWig file for visualization in the genome browsers more efficiently. A qualitative assessment of peak quality can be determined by static genome browser track views in the CHIPS output.

After peak calling, the fraction of reads in peaks (FRIP) scores is calculated to assess the samples' quality. The FRIP score is the fraction of 4,000,000 subsampled reads that fall within the peak regions. FRIP score increases with sequencing depth, so a subsample of reads is used. The FRIP score indicates data's signal-to-noise ratio, and a higher FRIP score indicates higher quality.

Certain characteristics of the peaks can be used to describe further the quality of the data. Peaks from a high-quality sample should have a high percentage of overlap with the known DNaseI sites. CHIPS overlaps the peaks with the union of the public DNaseI hypersensitive sites to determine the data's quality. Moreover, high-quality peaks tend to be evolutionarily conserved across species. CHIPS plots the conservation plot across all peaks. The conservation plots of transcription factors typically show a high focal point around the peak summits, while histone modifications show bimodal peaks with a dip in the center.

\section{Downstream analysis}

Peak annotation is performed to describe how the peaks distribute across the genome. Specifically, CHIPS determines the proportions of peaks that overlap with promoters, exons, introns, or intergenic regions. Motif identification is carried out using HOMER v4.11 (Heinz et al. 2010). The top 5,000 most significant peak summits (ranked by the MACS P-value) are used for motif analysis. Finally, to determine which genes may be regulated by the peaks, a regulatory potential score is calculated for each gene using an exponential decay model implemented in LISA (Qin et al. 2020). LISA calculates regulatory potential scores that represent the cumulative influence of nearby peaks associated with each gene.

\section{Output}

CHIPS provides results files in.txt and.png forms inside well-structured folders and a dynamic HTML report summarizing quality control metrics at the sample level. An example report for the Cancer Genome Atlas Lung Adenocarcinoma (TCGA-LUAD) ATAC-seq data is available at here.

\section{Operation}

CHIPS (Tang, 2021) can be executed in any Linux-based operating system. All the tools can be installed through Conda. Documentation accompanying the CHIPS software describes the installation process and the structure of the analysis 
results and report directories. Due to the modular nature of the Snakemake workflow, the report can be customized to meet individual needs and easily expanded if new metrics are added. Furthermore, the same metrics are reported in the CistromeDB (Zheng et al. 2019) which facilitates comparisons of results with that resource.

\section{Use case}

There are three steps to run CHIPS. Step1: Install CHIPS Conda environment and download reference files. Step2: Set up CHIPS project folder. Step3: Run the Snakemake pipeline. All work is done within a single project folder. Four core components are required within the project directory: CHIPS/, config.yaml, metasheet.csv, and ref_files/. Each core component is indispensable to run CHIPS, and their relative paths are restricted. Optionally, we recommend soft linking the data folder to the project directory. If the analysis uses data from species other than human or mouse, a ref.yaml file must also be attached to indicate where the references are. In the following examples, we will use human TCGA and ENCODE ATAC-seq data to illustrate how to run the CHIPS in detail.

\section{Use Case 1: Processing the Cancer Genome Atlas Lung Adenocarcinoma (TCGA-LUAD) ATAC-seq data} by CHIPS

In this use case, we will demonstrate how to set up and run a CHIPS pipeline using 22 TCGA ATAC-seq datasets with bam files as input. Data are available in Genomic Data Commons Data Portal (see Underlying data). A recommended way to download the data is to use the manifest file.

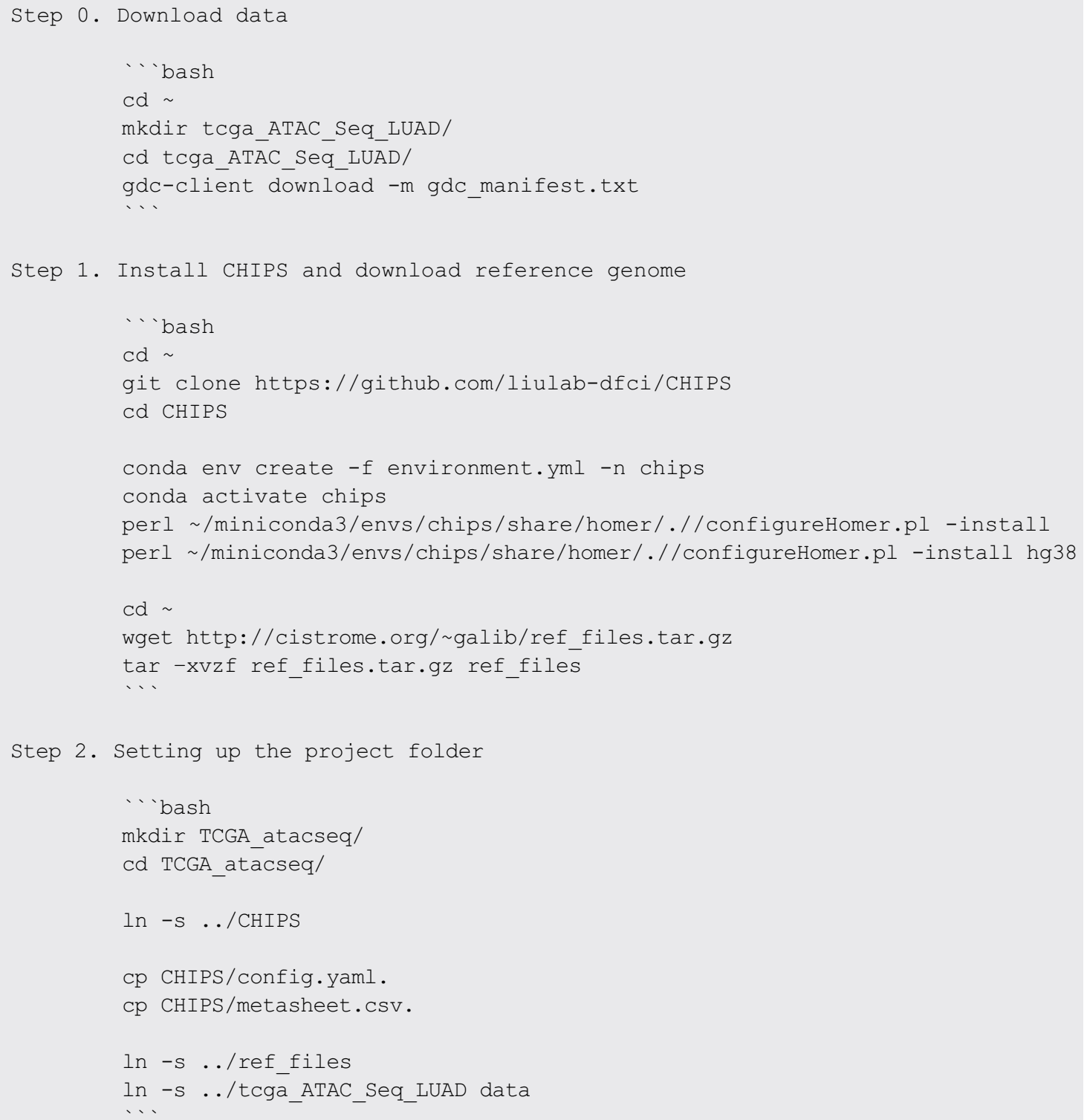




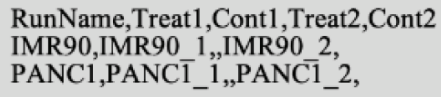

Figure 5. metasheet.csv file for processing ENCODE data

Then, we edit config,yaml to give each pair of fastq.gz files a sample name (Figure 4). In metasheet.csv file, we group replicates within the same run and leave control group empty (Figure 5).

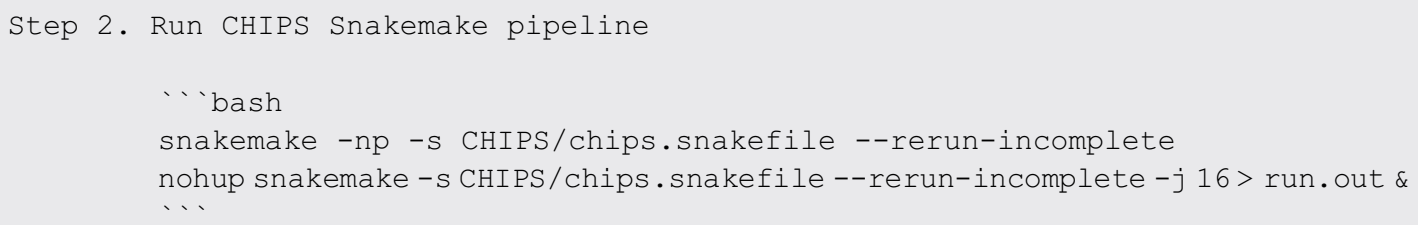

Check the run.out and when the pipeline prints out $100 \%$ complete, an analysis folder with all the output and report will be generated within the project directory.

\section{Conclusion}

Taken together, CHIPS is a scalable and reproducible pipeline written in Snakemake. It performs quality control and reproducible processing of the chromatin profiling data generated from ATAC-seq, ChIP-seq, and DNase-seq experiments. CHIPS does not explicitly label samples as being "low" or "high" quality overall. We rely on the users to interpret information from multiple quality control features to determine which samples to include for further downstream analyses. CHIPS also does not provide downstream analyses comparing cases and controls. Downstream analyses depend on the biological context of the experiments and may consist of differential binding, motif analysis, and pathway analysis in the setting of chromatin profiling experiments. An independent Snakemake pipeline COBRA (Qiu et al. 2020) is designed for this purpose.

Data availability

Underlying data

- The TCGA LUAD ATACseq data for Use Case 1 are available from Genomic Data Commons Data Portal: https://bit.ly/3bPytgG.

- The ENCODE ATACseq data for Use Case 2 are available from ENCODE data portal: https://www.encodeproject.org/experiments/ENCSR200OML/ (ATAC-seq of Panc1). https://www.encodeproject.org/experiments/ ENCSR591PIX/ (ATAC-seq of IMR-90).

Software availability

Source code available from: https://github.com/liulab-dfci/CHIPS.

Archived source code at time of publication: http://doi.org/10.5281/zenodo.4782801 (Tang, 2021).

License: MIT.

Acknowledgements

We thank the Center for Functional Cancer Epigenetics and Molecular Biology Core Facilities at Dana-Farber Cancer Institute for valuable feedback on CHIPS. 
Chen HX, Song M, Maecker HT, et al.: Network for Biomarker Immunoprofiling for Cancer Immunotherapy: Cancer Immune Monitoring and Analysis Centers and Cancer Immunologic Data Commons (CIMAC-CIDC). Clin Cancer Res: An Official Journal of the American Association for Cancer Research, January, clincanres. 2021; 3241: 2020. PubMed Abstract | Publisher Full Text

Chen S, Zhou Y, Chen Y, et al.: Fastp: An Ultra-Fast All-in-One FASTQ Preprocessor. Bioinformatics. 2018.

PubMed Abstract | Publisher Full Text | Free Full Text

Furey TS: ChIP-Seq and beyond: New and Improved Methodologies to Detect and Characterize Protein-DNA Interactions. Nat Rev Genet.

2012;

PubMed Abstract | Publisher Full Text | Free Full Text

Heinz S, Benner C, Spann N, et al.: Simple Combinations of LineageDetermining Transcription Factors Prime Cis-Regulatory Elements

Required for Macrophage and B Cell Identities. Mol Cell. 2010;

PubMed Abstract | Publisher Full Text | Free Full Text

Köster J, Rahmann S: Snakemake-a Scalable Bioinformatics Workflow Engine. Bioinformatics. 2012; 28(19): 2520-2522.

PubMed Abstract | Publisher Full Text

$\mathrm{Li} \mathrm{H}$ : Aligning Sequence Reads, Clone Sequences and Assembly Contigs with BWA-MEM. ArXiv [q-Bio.GN]. arXiv. 2013.

Reference Source
Meyer CA, Liu XS: Identifying and Mitigating Bias in Next-Generation Sequencing Methods for Chromatin Biology. Nat Rev Genet. 2014; 15(11): 709-721.

PubMed Abstract | Publisher Full Text | Free Full Text

Qin Q, Fan F, Zheng R, et al.: Lisa: Inferring Transcriptional Regulators through Integrative Modeling of Public Chromatin Accessibility and ChIP-Seq Data. Genome Biol. 2020: 21 (1): 32.

PubMed Abstract | Publisher Full Text | Free Full Text

Qiu X, Feit AS, Feiglin A, et al.: CoBRA: Containerized Bioinformatics Workflow for Reproducible ChIP/ATAC-Seq Analysis - from

Differential Peak Calling to Pathway Analysis. Cold Spring Harbor

Laboratory. 2020.

Publisher Full Text

Tang M: liulab-dfci/CHIPS: Release v1.0.0 (Version v1.0.0). Zenodo. 2021, May 24.

Publisher Full Text

The-ENCODE Project Consortium: An Integrated Encyclopedia of DNA Elements in the Human Genome. Nature. 2012; 489(7414): 57-74. PubMed Abstract | Publisher Full Text | Free Full Text

Zheng R, Changxin W, Shenglin M, et al.: Cistrome Data Browser: Expanded Datasets and New Tools for Gene Regulatory Analysis. Nucleic Acids Res. 2019; 47 (D1): D729-35.

PubMed Abstract | Publisher Full Text | Free Full Text 


\section{Open Peer Review}

\section{Current Peer Review Status: $\mathrm{X}$}

\section{Version 1}

Reviewer Report 16 November 2021

https://doi.org/10.5256/f1000research.56206.r98897

(C) 2021 Zhu A. This is an open access peer review report distributed under the terms of the Creative Commons Attribution License, which permits unrestricted use, distribution, and reproduction in any medium, provided the original work is properly cited.

\section{Anqi Zhu}

Genentech, Inc., San Francisco, CA, USA

In this manuscript, Taing et al. introduced a pipeline that they developed using the Snakemake software to streamline the steps of processing chromatin profiling data, which includes the raw data processing, quality control and downstream analysis tasks. The pipeline is useful, however the manuscript could benefit from adding more description and discussion of the innovation and implementation details of the pipeline. The detailed comments are as below.

1. What are the needs to develop the proposed pipeline after investigating the existing pipelines that process and analyze the chromatin profiling data? What are the differences between the developed pipeline and the existing pipelines that benefit the users?

2. In the introduction, the authors discussed the importance of removing the bias and the reproducibility of the data processing. The authors could highlight the steps where such goals are accomplished.

3. How were the softwares integrated into this pipeline. E.g: any specific steps needed to make sure the output/input files are compatible, or to reduce I/O burden?

4. Is batch correction step part of the pipeline? If not, can you briefly explain the reason?

5. What are the reasons of picking these selected softwares/packages in the pipeline? Can you briefly describe the methods, and comment on them?

6. In the use cases, I would be more interested to read how differently (or more efficiently) CHIPS handles the data from the existing pipelines, and the interpretation of the outputs from CHIPS with these two data, rather than how to set up and run the pipeline, which are also super important, but more suitable for a README or vignette but not a publication. Some minor things:

1. "In addition, $4,000,000$ reads are down sampled for calculating the PCR bottleneck coefficient (PBC)" It's not clear to me where does the 4,000,000 reads sampled from? 
2. Some of the bash codes could be removed, so that the manuscript is more succinct.

3. It can be a little confusing to the reader that in the conclusion, it is said "CHIPS also does not provide downstream analyses comparing cases and controls." While I understand this is not the same set of downstream analysis as provided in CHIPS, you could make it a little clearer.

Is the rationale for developing the new software tool clearly explained?

Partly

Is the description of the software tool technically sound?

Partly

Are sufficient details of the code, methods and analysis (if applicable) provided to allow replication of the software development and its use by others?

Partly

Is sufficient information provided to allow interpretation of the expected output datasets and any results generated using the tool?

Partly

Are the conclusions about the tool and its performance adequately supported by the findings presented in the article?

No

Competing Interests: No competing interests were disclosed.

Reviewer Expertise: Statistical genomics, Bulk and single-cell RNA-seq data, Statistical software development

I confirm that I have read this submission and believe that I have an appropriate level of expertise to state that I do not consider it to be of an acceptable scientific standard, for reasons outlined above. 
The benefits of publishing with F1000Research:

- Your article is published within days, with no editorial bias

- You can publish traditional articles, null/negative results, case reports, data notes and more

- The peer review process is transparent and collaborative

- Your article is indexed in PubMed after passing peer review

- Dedicated customer support at every stage

For pre-submission enquiries, contact research@f1000.com 